\title{
Índice de Paridad Poder de Compra Nutricional: comparación de los precios calóricos entre dietas habituales y saludables
}

\author{
Alfonso Mendoza Velázquez ${ }^{1}$
}

Forma de citar

Mendoza Velázquez A. Índice de Paridad Poder de Compra Nutricional: comparación de los precios calóricos entre dietas habituales y saludables. Rev Panam Salud Publica. 2012;31(1)17-24.

RESUMEN Objetivo. Validar el Índice de Paridad Poder de Compra Nutricional (Nut3-CiO) como un instrumento que permite comparar los precios calóricos de una dieta saludable versus una dieta no saludable entre regiones o ciudades de un país a través del tiempo.

Métodos. Se construyeron índices de precios calóricos y se empleó la "ley de un solo precio" para derivar el índice Nut3-CiO. Se obtuvieron tasas de inflación calóricas sobre las que se realizó estadística descriptiva básica. El Nut3-CiO se aplicó a las principales ciudades de México durante el período enero de 1996 a diciembre 2010.

Resultados. El comportamiento estadístico del Nut3-CiO reveló que en las ciudades de México los productos de la dieta habitual son más baratos que los productos de la dieta saludable. Se observó un comportamiento cíclico del índice, una correlación alta entre la inflación de la dieta habitual y la inflación de la canasta básica, y una alta persistencia de los precios.

Conclusiones. El índice Nut3-CiO permite comparar periódicamente el diferencial de precios de dos tipos de dietas — habituales y saludables - entre las ciudades de un mismo país. Este instrumento podría ayudar a las autoridades de salud a identificar las ciudades en donde resulta más —o menos-fácil para los consumidores acceder a una dieta habitual o saludable en términos de costo. Asimismo, hace posible estimar el porcentaje de ajuste que deberían tener los precios relativos en cada ciudad para alcanzar los niveles de paridad poder de compra nutricional.

Palabras clave Obesidad; sobrepeso; dieta; conducta alimentaria; alimentos; economía de la salud; indicadores económicos; bioestadística; México.

La obesidad, cuya prevalencia global se ha más que duplicado desde 1980, está en camino de convertirse en el enemigo de salud pública número uno en el mundo $(1,2)$. Según datos de la Organización Mundial de la Salud, en 2008 alrededor de 1500 millones de

Universidad Popular Autónoma del Estado de Puebla, Centro de Investigación e Inteligencia Económica (CIIE), Puebla, México. La correspondencia se debe dirigir a Alfonso Mendoza Velázquez. Correo electrónico: alfonso.mendoza@upaep.mx; amv101@yahoo.com personas de 20 años de edad o mayores tenían sobrepeso, de las cuales más de 200 millones de hombres y cerca de 300 millones de mujeres eran obesas. En 2010, aproximadamente 43 millones de niños menores de 5 años registraban sobrepeso (1).

El sobrepeso y la obesidad son efectivamente fenómenos sociales que, además de representar un reto médico, generan una carga financiera significativa para el Estado y para las propias personas que los padecen. En este sentido, hoy por hoy se reconoce ampliamente que la obesidad constituye un problema multifactorial no solo determinado por variables médicas, sociales y conductuales, sino también asociado a las condiciones económicas. Sin embargo, aun cuando se ha observado la importancia que revisten los determinantes económicos de la obesidad, hasta el momento este tema ha sido quizá el menos explorado en la literatura científica (3-5).

Hay no obstante unos pocos estudios que han arrojado algo de luz en relación con el impacto del nivel económico en la obesidad, en particular relacionados con 
los precios de los alimentos saludables y los no saludables. Se ha encontrado, por ejemplo, que en Estados Unidos los precios de los alimentos saludables suben más rápido que los de los alimentos en general (6). Tal realidad podría estar desplazando a las dietas saludables fuera del alcance de los bolsillos de muchos habitantes de ese país, quienes estarían así consumiendo menos alimentos nutritivos, como frutas y hortalizas, que resultan más caros que los productos caracterizados como "dietas densas", con mayores contenidos de azúcares, grasas y granos refinados.

Uno de estos trabajos, centrado específicamente en el impacto que tienen los precios en la elección de los alimentos, halló que los productos de alta densidad energética son más baratos y más resistentes al efecto inflacionario, lo que podría explicar en parte las mayores tasas de obesidad observadas entre los grupos de menores ingresos dentro de la población estadounidense. Como se dijo, los productos de baja densidad calórica registran incrementos de precios significativamente mayores al promedio de los alimentos en general, lo que podría actuar como una barrera económica a la adopción de dietas más sanas (6).

En Australia, una investigación reciente encontró que los precios de algunos alimentos - como las frutas y los vegetales- subieron drásticamente entre 2000 y 2007, mientras que los de otros, con alto contenido calórico, han incluso bajado durante el mismo período (7). Ese estudio da seguimiento y monitorea la accesibilidad a una canasta de 57 productos sanos para una familia de cinco integrantes. Sin embargo, la principal dificultad que enfrenta es que se examina una sola región y solo tres años irregulares: 2000, 2005 y 2007.

En la actualidad no hay un instrumento de política pública que permita dar seguimiento periódico consistente, confiable y oportuno al comportamiento de los precios de productos saludables y no saludables entre las regiones o ciudades de un país. Los índices de precios de productos saludables y no saludables introducidos hasta el momento se han limitado a examinar una sola región o un solo país, y en un lapso específico, sin llevar a cabo una comparación periódica entre los precios de alimentos sanos y no sanos entre ciudades o países. Esta omisión también es característica de algunos índices de precios, de índices de calidad nutricional y de accesibilidad de los alimentos (8-11).

El grave problema del sobrepeso requiere de instrumentos objetivos para examinar de manera periódica la asociación entre obesidad y los precios de productos saludables y no saludables. También es perentorio determinar, no solo a nivel país sino a nivel regional o entre ciudades, si los precios relativos de los alimentos saludables y no saludables guardan alguna relación con desórdenes o trastornos alimentarios. Desafortunadamente, las herramientas de precios que hay disponibles para hacer este tipo de comparaciones - p. ej. determinar la brecha de precios en productos saludables versus no saludables o comprender la transición nutricional de la población por regiones - no son lo suficientemente idóneas.

El objetivo del presente estudio fue precisamente validar el nuevo Índice de Paridad Poder de Compra Nutricional (Nut3-CiO), como un instrumento que permite comparar los precios calóricos de una dieta saludable versus una dieta no saludable, entre regiones o ciudades de un país a través del tiempo, basados en la Ley de un Solo Precio (12).

\section{MÉTODO}

Para validar el índice Nut3-CiO se calcularon primero los "índices de precios calóricos" (IPC) asociados a una dieta habitual y a una dieta saludable en México. La canasta de productos para definir la dieta habitual se extrajo del gasto diario por producto que los mexicanos reportaron en la Encuesta Nacional de Ingreso y Gasto de los Hogares 2008 (ENIGH) del Instituto Nacional de Estadística y Geografía (INEGI). Si bien el gasto promedio diario por producto no necesariamente tiene que ser igual al consumo diario, es de hecho la variable disponible más aproximada porque proporciona una idea clara del perfil de consumo diario del mexicano. El primer cuadro del material suplementario del presente trabajo muestra el consumo calórico promedio diario de los mexicanos por producto. La participación del consumo calórico por producto $\left(q_{i, 0}\right)$ define el perfil de consumo habitual y ofrece un ponderador para cada uno de los productos usados como insumos del IPC en la dieta habitual.

Por su parte, la canasta de consumo saludable se basa en la dieta propuesta por Pérez Lizaur para un varón mexicano promedio (13). El cuadro 1 muestra la guía alimentaria de la dieta saludable ajustada por alimentos equivalentes y por componentes: calorías, hidratos de carbono, lípidos y proteínas. ${ }^{2}$ El diseño nutricional presentado en esta dieta contiene ingredientes que en conjunto conforman una canasta de productos saludables. Para la construcción del IPC de la dieta saludable se tomó como ponderador la proporción de calorías respecto al total por ingrediente (cuadro 2).

La muestra en este estudio estuvo conformada por los índices de precios de los alimentos listados en el material suplementario y en el cuadro 1 que el INEGI calcula periódicamente para 46 ciudades de México, desde enero de 1995 a diciembre de 2010, con período base febrero de 2001. Para contrastar también se obtuvieron los índices de precios de la canasta básica (CBA), la CBA calórica y el Índice Nacional de Precios al Consumidor (INPC).

\section{Índices de precios calóricos}

Los insumos fundamentales del índice Nut3- $-\mathrm{CiO}_{j, t}$ son los índices de precios nutricionales o calóricos construidos a partir del método de Laspeyres, el cual mantiene fijas las proporciones de calorías aportadas por cada ingrediente pero permite la variación temporal de los precios de alimentos individuales. El IPC de la dieta saludable se calcula con $P_{s, t}=$ $\left(\Sigma p_{i, t} q_{i, 0}\right) /\left(\Sigma p_{i, 0} q_{i, 0}\right)$ y el de la dieta habitual con $P_{h, t}=\left(\Sigma p_{i, t} q_{i, 0}\right) /\left(\Sigma p_{i, 0} q_{i, 0}\right)$, donde los subíndices $s$ y $h$ se refieren a las canastas saludable y habitual, respectivamente; $p_{i, t}$ es el índice de precios del ingrediente $i$ en el período $t$; $p_{i, 0}$ es el precio por ingrediente en el período base $(t=0)$ y $q_{i, 0}$ es la proporción de calorías en el período base para cada canasta, respectivamente. La construcción de estos índices calóricos es especialmente viable cuando el analista cuenta con mediciones de con-

\footnotetext{
2 Los equivalentes elegidos en esta dieta saludable son similares a los valores nutrimentales de referencia para la población mexicana descritos en Bourges-Rodríguez y colaboradores (15). Una comparación entre las dos dietas mostró que los carbohidratos y las proteínas de la dieta saludable en este estudio están por arriba de los valores máximos recomendados en apenas $2 \%$ y 3,5\% respectivamente, mientras que los lípidos están 9 puntos porcentuales por debajo del mínimo recomendado. Estas diferencias se explican por el uso de equivalentes compatibles con los alimentos a los que da segumiento periódicamente el Instituto Nacional de Estadística y Geografía (INEGI).
} 
CUADRO 1. Alimentos que componen una dieta saludable en México

\begin{tabular}{|c|c|c|c|c|c|c|}
\hline Alimento & Porción & Equivalente $^{\mathrm{a}}$ & Kcal & $P$ & $\mathrm{~L}$ & $\mathrm{HC}$ \\
\hline \multicolumn{7}{|l|}{ Desayuno } \\
\hline Jugo de naranja & Un vaso chico & Una fruta & 60,0 & 0,0 & 0,0 & 15,0 \\
\hline Papaya picada & Una taza & Una fruta & 60,0 & 0,0 & 0,0 & 15,0 \\
\hline Huevo a la mexicana & Dos unidades & $\begin{array}{l}\text { Dos alimentos de origen animal con moderación, } \\
\text { verdura y una porción de grasa }\end{array}$ & 206,9 & 14,5 & 15,1 & 2,9 \\
\hline Tortillas & Dos unidades & Dos porciones moderadas de cereal & 140,0 & 4,0 & 0,0 & 30,0 \\
\hline Café con azúcar & Una taza & Una porción moderada de azúcar & 40,0 & 0,0 & 0,0 & 10,0 \\
\hline \multicolumn{7}{|l|}{ Colasión } \\
\hline Zanahoria rallada con limón & Una taza & Verdura & 25,0 & 2,0 & 0,0 & 4,0 \\
\hline Galletas integrales & Cuatro unidades & Una porción de cereal & 70,0 & 2,0 & 0,0 & 15,0 \\
\hline \multicolumn{7}{|l|}{ Almuerzo } \\
\hline Sopa de verduras & Un tazón & $\begin{array}{l}\text { Una porción de verdura y una porción de grasa } \\
\text { moderada }\end{array}$ & 82,7 & 2,7 & 5,3 & 6,4 \\
\hline Salpicón & Un plato & $\begin{array}{l}\text { Dos porciones de carne baja en grasa, dos verduras } \\
\text { y dos porciones de grasa moderadas }\end{array}$ & 334,7 & 22,4 & 19,2 & 6,7 \\
\hline Bolillo sin migajón & Una unidad & Dos porciones de cereal & 183,0 & 5,7 & 0,0 & 38,4 \\
\hline Agua de Jamaica con azúcar & Dos tazas & Una porción moderada de azúcar & 80,0 & 0,0 & 0,0 & 20,0 \\
\hline Mandarina & Una unidad & Una fruta & 60,0 & 0,0 & 0,0 & 15,0 \\
\hline Café con azúcar & Una taza & Una porción moderada de azúcar & 40,0 & 0,0 & 0,0 & 10,0 \\
\hline \multicolumn{7}{|l|}{ Colasión } \\
\hline Agua natural & Dos tazas & & 0,0 & 0,0 & 0,0 & 0,0 \\
\hline \multicolumn{7}{|l|}{ Cena } \\
\hline Melón & Una rebanada & Una fruta & 60,0 & 0,0 & 0,0 & 15,0 \\
\hline Enfrijoladas con queso y crema & Dos unidades & $\begin{array}{l}\text { Dos porciones de cereal, una leguminosa } \\
\text { y dos porciones de grasa }\end{array}$ & 357,7 & 12,2 & 21,0 & 36,8 \\
\hline Jitomate rebanado & Una unidad & Una verdura & 11,0 & 0,6 & 0,1 & 2,4 \\
\hline Salsa verde & Media taza & Una verdura & 32,9 & 2,0 & 0,0 & 4,0 \\
\hline Pan dulce & Una unidad & Tres porciones de cereal con grasa moderada & 72,5 & 3,0 & 7,5 & 22,5 \\
\hline Leche con café & Una taza & Una leche descremada & 95,0 & 9,0 & 2,0 & 12,0 \\
\hline Totales & & & 2011,4 & 79,97 & 70,3 & 281,2 \\
\hline
\end{tabular}

Fuente: elaboración del autor, con base en (13-15). Se buscó mantener la consistencia con los índices de precios por ciudad que publica el Banco de México (http://www.banxico. org.mx) en la sección de estadísticas e inflación. Desde julio de 2011 la actualización de los precios está a cargo del Instituto Nacional de Estadística y Geografía (INEGI) de México (http://www.inegi.gob.mx).

Nota: Kcal: kilocalorías; P: proteínas; L: lípidos; HC; hidratos de carbono.

a Es un alimento que puede sustituir al propuesto en la primera columna por ser del mismo grupo nutrimental y tener una aportación calórica igual o muy similar.

sumo de alimentos poco frecuentes, pero con precios de alimentos actualizados periódicamente.

\section{La paridad poder de compra y el índice Nut3-CiO}

El índice Nut3-CiO tiene su origen en un concepto popular de la economía con el que se explica la diferencia relativa de los precios entre regiones a partir de la denominada paridad poder de compra (PPC), partiendo de la hipótesis de que en un mercado eficiente y $\sin$ restricciones el precio de una misma canasta de bienes en dos ciudades no debe ser distinto $(12,16)$. El índice Nut3-CiO usa como insumos dos precios calóricos relativos, uno para la canasta de productos saludables y otro para la canasta de productos habituales, denominados $P_{s, t} / P_{s, t}^{*}$ y $P_{h, t} / P_{h, t}^{*}$ respectivamente, donde $P^{*}$ denota el precio de la ciudad de referencia. Sus resultados se obtienen de la razón de precios relativos entre las dos dietas:

$$
\text { Nut3-CiO }{ }_{j, t}=\left[\left(P_{h, t} / P_{h, t}^{*}\right) /\left(P_{s, t} / P_{s, t}^{*}\right)\right],
$$

en una ciudad $j$ específica y para un período de tiempo $t$ determinado. El índice Nut3- $\mathrm{CiO}_{j, t}$ introducido en la ecuación [1] mide la diferencia relativa de los precios de la canasta saludable contra los precios de la canasta habitual en una ciudad determinada.

En términos porcentuales, el índice Nut3-CiO ${ }_{j, t}$ puede tomar los valores:

$$
\begin{array}{rll} 
& >0 & \text { Sobrevaluación } \\
\text { Nut3-CiO }_{j, t} & =0 & \text { Equilibrio } \\
& <0 & \text { Subvaluación. }
\end{array}
$$

Ciudades con Nut3-CiO ${ }_{j, t}>0$ (p. ej. márgenes de sobrevaluación) muestran que los precios relativos de la dieta habitual, la de alto contenido calórico, están por arriba de los precios relativos de la canasta saludable. Un margen de sobrevaluación en una ciudad describe una situación favorable, debido a que la dieta habitual es más cara y, de acuerdo con la ley de la demanda, los habitantes de esta ciudad deberían demandar más productos de la dieta sana. Por su parte, un margen de subvaluación indica que los precios relativos de la dieta habitual están por debajo de los precios de la dieta saludable. Esta sería una situación poco favorable para los habitantes de una ciudad particular, ya que los precios de la canasta saludable serían más altos $\mathrm{y}$, por tanto, podría estarse incentivando el consumo de canastas habituales, que podrían estar induciendo el sobrepeso. Así, mientras más se aleje esta ciudad del equilibrio (por debajo de cero), peores serán las condiciones de precios para seguir una dieta saludable. El margen de subvaluación indica el porcentaje en el que puede subir el precio de la canasta habitual (o bajar el de la saludable) para 
CUADRO 2. Aportación calórica de una canasta saludable, por ingrediente, México

\begin{tabular}{|c|c|c|c|}
\hline \multirow[b]{2}{*}{ Ingrediente ${ }^{a}$} & \multirow[b]{2}{*}{$\mathrm{Kcal}^{\mathrm{b}}(n)$} & \multicolumn{2}{|c|}{ Aportación (\%) } \\
\hline & & Por ingrediente ${ }^{c} q_{i, 0}$ & Acumulada $^{\mathrm{d}} \Sigma q_{i, 0}$ \\
\hline Tortilla & 280,0 & 13,92 & 13,92 \\
\hline Pan dulce & 72,5 & 3,60 & 17,53 \\
\hline Pan blanco & 183,0 & 9,10 & 26,63 \\
\hline Otras galletas (integrales) & 70,0 & 3,48 & 30,10 \\
\hline Carne de res (bistec, puntas) & 225,0 & 11,19 & 41,29 \\
\hline Leche & 95,0 & 4,72 & 46,01 \\
\hline Queso & 7,7 & 0,38 & 46,39 \\
\hline Crema & 45,0 & 2,24 & 48,63 \\
\hline Huevo & 150,0 & 7,46 & 56,09 \\
\hline Aceite & 213,0 & 10,59 & 66,68 \\
\hline Naranja o mandarina & 120,0 & 5,97 & 72,65 \\
\hline Papaya & 60,0 & 2,98 & 75,63 \\
\hline Limón & 0,0 & 0,00 & 75,63 \\
\hline Melón & 60,0 & 2,98 & 78,61 \\
\hline Jitomate & 21,0 & 1,04 & 79,66 \\
\hline Cebolla & 23,0 & 1,14 & 80,80 \\
\hline Tomate & 21,0 & 1,04 & 81,84 \\
\hline Calabacita & 21,0 & 1,04 & 82,89 \\
\hline Chile serrano & 8,5 & 0,42 & 83,31 \\
\hline Zanahoria & 25,0 & 1,24 & 84,55 \\
\hline Ejote & 22,0 & 1,09 & 85,65 \\
\hline Frijoles & 120,0 & 5,97 & 91,62 \\
\hline Azúcar & 160,0 & 7,95 & 99,57 \\
\hline Café & 0,0 & 0,00 & 99,57 \\
\hline Caldo de pollo & 5,0 & 0,25 & 99,81 \\
\hline Cilantro, perejil, etc. & 3,7 & 0,18 & 100,00 \\
\hline \multicolumn{4}{|c|}{$\begin{array}{l}\text { Fuente: elaboración del autor con base en base el cuadro } 1 \text { y }(13,14,23) \text {. } \\
\text { a Los alimentos integrados por más de un ingrediente en el cuadro } 1 \text { se descomponen para obtener esta lista de } \\
\text { ingredientes individuales. } \\
\text { b El número de calorías corresponde a la cantidad consumida total de cada ingrediente según el cuadro } 1 . \\
\text { c Este número representa la proporción porcentual de calorías aportada por cada ingrediente respecto al total en la } \\
\text { dieta saludable definida en el cuadro } 1 . \\
\text { d Se presenta el porcentaje acumulado de calorías por cada ingrediente de acuerdo al orden definido en la primera } \\
\text { columna. }\end{array}$} \\
\hline
\end{tabular}

alcanzar la paridad de precios. Finalmente, una situación de equilibrio se obtiene cuando la relación de precios de la canasta habitual es igual a la relación de precios de la canasta saludable, es decir que los precios relativos de las dos dietas son iguales, significando que en esa ciudad comer saludable y seguir el consumo habitual tendrían el mismo costo.

\section{Análisis estadístico}

Se utilizaron diversas medidas de tendencia central, dispersión y correlación para examinar el comportamiento de las series de precios calóricos de la dieta habitual y la saludable. Se estimaron coeficentes de correlación lineal entre las series temporales de precios para cada ciudad con el índice de precios de la CBA y el INPC. A partir del análisis descriptivo (medidas de dispersión y tendencia central), se eligió la ciudad numeraria y se calculó el índice Nut3$\mathrm{CiO}$. La ciudad numeraria o de referencia es la ciudad donde los precios han sido más estables y bajos históricamente. Finalmente se determinó la distribución cuantílica mensual de Nut3-CiO para todo el período de estudio.

\section{RESULTADOS}

\section{La canasta saludable y la ciudad numeraria}

Los cuadros del material suplementario describen las tasas de inflación calórica anuales calculadas a partir del índice de precios saludables $P_{s, t^{*}}$. Examinando la media muestral, la desviación estándar y el coeficiente de variación, se halló que la ciudad con el menor crecimiento promedio y la menor dispersión inflacionaria en el período enero 1996diciembre de 2010 fue Iguala, en el estado de Guerrero. El coeficiente de autocorrelación de la inflación calórica en un mes determinado respecto al mismo mes de un año anterior, denotado por $\rho_{t, t-12}$ indicó que esa ciudad, además de tener niveles de inflación bajos, estos tendieron a mantenerse así por más tiempo, razón por la cual Iguala fue elegida la ciudad numeraria y sus precios calóricos se expresaron como $P_{h, t}^{*}$ y $P_{s, t}^{*}$ para la dieta habitual y la saludable, respectivamente.

\section{La canasta habitual}

De todas las ciudades consideradas, Tepic (Nayarit) fue la que tuvo la inflación promedio anual más baja $(9,85 \%)$, posicionándola como la ciudad del país donde a diciembre de 2010 fue más fácil acceder a la dieta habitual del mexicano. Cerca de esta condición se encontraron otras ciudades como Tlaxcala, Tehuantepec y Guadalajara, con tasas promedio anuales de 10,59\%, 10,39\% y 10,32\%, respectivamente. Por otro lado, las ciudades en donde fue más caro acceder a una dieta de alto contenido calórico se ubicaron generalmente en el norte del país. La ciudad con los precios de la dieta habitual más altos fue Hermosillo (Sonora), donde se registró un crecimiento promedio anual de 11,66\%, seguido de cerca por Chihuahua, La Paz y Tijuana, con tasas de $11,41 \%, 11,37 \%$ y $11,32 \%$, respectivamente. Se observó que la persistencia de la inflación de la canasta habitual fue más alta que la de la canasta saludable. Además, la inflación calórica de las dos dietas tuvo una mayor correlación con la inflación de la CBA que con la del INPC.

\section{El Índice de Paridad Poder de Compra Nutricional}

La aplicación del índice Nut3- $\mathrm{C}_{\mathrm{i}} \mathrm{O}$ definido en la ecuación [1] para el caso mexicano permitió examinar el comportamiento relativo de los precios por ciudad, así como las características de acceso a dietas saludables y habituales en términos de precios. En el cuadro 3 se muestran los valores de los índices de precios nutricionales de las canastas habitual y saludable $\left(P_{h, t}\right.$ y $\left.P_{s, t}\right)$ a diciembre de 2010 y para cada ciudad en México. De acuerdo con el índice de la dieta habitual $\left(P_{h, t}\right)$, las ciudades donde resultó más caro adquirir una dieta habitual, alta en contenido calórico, fueron Veracruz, Cuernavaca y Hermosillo, mientras que las ciudades donde resultó más barato consumir estas dietas fueron Tijuana, Guadalajara y Tlaxcala. Por otro lado, en Ciudad Jiménez, Iguala y Tepatitlán las dietas saludables fueron las 
CUADRO 3. Índice de Paridad Poder de Compra Nutricional (Nut3-CiO), México, diciembre de 2010

\begin{tabular}{|c|c|c|c|c|c|c|}
\hline & \multicolumn{2}{|c|}{ Nivel de precios $\left(P_{d, t}\right)^{\mathrm{a}}$} & \multicolumn{2}{|c|}{ Precios relativos (\%) } & \multicolumn{2}{|c|}{ Índice Nut3-CiO } \\
\hline & $P_{h, t}$ & $P_{s, t}$ & $P_{h, t} / P_{h, t}^{*}$ & $P_{s, t} / P_{s, t}^{*}$ & Margen $(\%)^{b}$ & Posición \\
\hline Iguala, Guerrero & 165,3 & 168,7 & 0 & 0 & 0 & 1 \\
\hline Tepatitlán, Jalisco & 195,8 & 170,8 & 18,5 & 1,2 & 17,1 & 2 \\
\hline Ciudad Jiménez, Chihuahua & 183,7 & 165,3 & 11,1 & $-2,0$ & 13,4 & 3 \\
\hline Campeche, Campeche & 180,6 & 174,5 & 9,3 & 3,4 & 5,7 & 4 \\
\hline Tlaxcala, Tlaxcala & 179,3 & 175,2 & 8,5 & 3,9 & 4,5 & 5 \\
\hline Chihuahua, Chihuahua & 185,1 & 181,0 & 12,0 & 7,3 & 4,4 & 6 \\
\hline Acapulco, Guerrero & 185,6 & 181,5 & 12,3 & 7,6 & 4,4 & 7 \\
\hline Hermosillo, Sonora & 205,5 & 201,0 & 24,3 & 19,2 & 4,3 & 8 \\
\hline Mexicali, Baja California & 179,5 & 176,3 & 8,5 & 4,5 & 3,9 & 9 \\
\hline Veracruz, Veracruz & 213,7 & 210,9 & 29,3 & 25,0 & 3,4 & 10 \\
\hline León, Guanajuato & 180,1 & 179,8 & 8,9 & 6,6 & 2,2 & 11 \\
\hline Monclova, Coahuila & 184,4 & 184,6 & 11,6 & 9,4 & 2,0 & 12 \\
\hline Morelia, Michoacán & 185,6 & 185,9 & 12,4 & 10,2 & 1,9 & 13 \\
\hline Mérida, Yucatán & 198,1 & 198,3 & 19,9 & 17,6 & 1,9 & 14 \\
\hline Tehuantepec, Oaxaca & 200,3 & 200,8 & 21,2 & 19,0 & 1,9 & 15 \\
\hline Cuernavaca, Morelos & 206,9 & 207,6 & 25,2 & 23,1 & 1,7 & 16 \\
\hline San Luis Potosí, San Luis Potosí & 184,3 & 185,2 & 11,5 & 9,8 & 1,6 & 17 \\
\hline Ciudad Juárez, Chihuahua & 183,5 & 185,4 & 11,0 & 9,9 & 1,0 & 18 \\
\hline Chetumal, Quintana Roo & 189,3 & 191,5 & 14,6 & 13,5 & 0,9 & 19 \\
\hline Aguascalientes, Aguascalientes & 190,8 & 193,1 & 15,4 & 14,5 & 0,8 & 20 \\
\hline Guadalajara, Jalisco & 172,1 & 174,6 & 4,2 & 3,5 & 0,6 & 21 \\
\hline Durango, Durango & 188,6 & 191,3 & 14,1 & 13,4 & 0,6 & 22 \\
\hline La Paz, Baja California Sur & 195,9 & 198,9 & 18,5 & 17,9 & 0,5 & 23 \\
\hline Villahermosa, Tabasco & 198,7 & 202,9 & 20,2 & 20,3 & $-0,1$ & 24 \\
\hline Tulancingo, Hidalgo & 184,8 & 189,5 & 11,8 & 12,3 & $-0,4$ & 25 \\
\hline Oaxaca, Oaxaca & 183,4 & 188,6 & 10,9 & 11,8 & $-0,7$ & 26 \\
\hline Colima, Colima & 193,3 & 198,8 & 16,9 & 17,8 & $-0,8$ & 27 \\
\hline Córdoba, Veracruz & 188,6 & 194,5 & 14,1 & 15,3 & $-1,0$ & 28 \\
\hline Huatabampo, Sonora & 185,4 & 191,8 & 12,2 & 13,7 & $-1,3$ & 29 \\
\hline Cortázar, Guanajuato & 189,4 & 196,1 & 14,6 & 16,3 & $-1,4$ & 30 \\
\hline Querétaro, Querétaro & 190,6 & 197,3 & 15,3 & 16,9 & $-1,4$ & 31 \\
\hline Jacona, Michoacán & 187,5 & 195,0 & 13,5 & 15,6 & $-1,9$ & 32 \\
\hline Culiacán, Sinaloa & 196,5 & 204,5 & 18,9 & 21,2 & $-1,9$ & 33 \\
\hline Toluca, México & 180,2 & 188,0 & 9,0 & 11,5 & $-2,2$ & 34 \\
\hline Matamoros, Tamaulipas & 193,1 & 202,2 & 16,8 & 19,8 & $-2,5$ & 35 \\
\hline Monterrey, Nuevo León & 190,4 & 199,6 & 15,2 & 18,3 & $-2,6$ & 36 \\
\hline Fresnillo, Zacatecas & 183,2 & 192,0 & 10,8 & 13,8 & $-2,6$ & 37 \\
\hline Tampico, Tamaulipas & 200,7 & 212,0 & 21,4 & 25,7 & $-3,4$ & 38 \\
\hline Torreón, Coahuila & 197,0 & 208,3 & 19,2 & 23,5 & $-3,4$ & 39 \\
\hline Tapachula, Chiapas & 185,7 & 196,6 & 12,3 & 16,5 & $-3,6$ & 40 \\
\hline Tepic, Nayarit & 191,0 & 203,0 & 15,6 & 20,3 & $-4,0$ & 41 \\
\hline Área Metropolitana, D.F. & 191,5 & 205,3 & 15,9 & 21,7 & $-4,8$ & 42 \\
\hline Puebla, Puebla & 184,4 & 197,8 & 11,6 & 17,2 & $-4,8$ & 43 \\
\hline Tijuana, Baja California & 167,3 & 179,8 & 1,3 & 6,6 & $-5,0$ & 44 \\
\hline San Andrés Tuxtla, Veracruz & 188,0 & 204,4 & 13,8 & 21,2 & $-6,1$ & 45 \\
\hline Cuidad Acuña, Coahuila & 183,3 & 201,8 & 10,9 & 19,7 & $-7,3$ & 46 \\
\hline
\end{tabular}

Fuente: elaboración del autor.

a Índice de precios a junio de 2010 con período base febrero de 2001. $P_{h, t}$ se refiere al índice de precios de la dieta habitual y $P_{s, t}$ se refiere al índice de precios de la dieta saludable, ambos calculados con la fórmula Laspeyres especificada en la subsección índices de precios calóricos. Para el cálculo de cada índice se empleó la información calórica de los ingredientes del cuadro 2 y del material suplementario para la dieta saludable y habitual, respectivamente. Estas dos columnas reportan índices que miden el comportamiento de los precios, ponderado por las calorías en cada dieta, por lo que no están expresados en pesos mexicanos, sino en puntos.

b Indica el margen de sobrevaluación o subvaluación relativo entre los precios de la dieta habitual y los precios de la dieta saludable definido en la ecuación [2].

más baratas, mientras que las más caras fueron en Tampico, Veracruz y Torreón.

Asimismo, en el cuadro 3 se pueden ver los precios relativos de las dietas habitual y saludable $\left(P_{h t} / P_{h, t}^{*}\right.$ y $\left.P_{s t} / P_{s t}^{*}\right)$ respecto a los valores de la ciudad numeraria. Tal como se dijo, mientras más se acercan a cero los valores de una ciudad determinada, mayor es la convergencia de precios para cada dieta entre esa ciudad y la ciudad de referencia.
Como se observa en el cuadro, Tijuana presentó una mayor convergencia con Iguala en la dieta habitual, mientras que Tepatitlán lo hizo en la dieta saludable. Finalmente, en la última columna del cuadro se muestra el margen de sobrevaluación nutricional obtenido mediante el índice Nut3-CiO e introducido en la fórmula [1]. Un margen positivo resulta favorable, ya que indica que los precios relativos de la dieta habitual en esa ciudad son relativamente más altos, haciendo que los habitantes se vean motivados a consumir dietas más saludables y relativamente más baratas (ver también las columnas 4 y 5 del cuadro 3). En esta situación favorable se encontraron -a diciembre de 2010- 23 de las 46 ciudades estudiadas, encabezadas por Tepatitlán, Ciudad Jiménez y Campeche, con porcentajes de 17,05, 13,41 y 5,65 , respectivamente. 
Por otro lado, las tres ciudades más desfavorecidas en la relación de precios entre dietas habituales y dietas saludables fueron Ciudad Acuña, San Andrés Tuxtla y Tijuana, con valores de $-7,29 \%,-6,12 \%$ y $-5 \%$, respectivamente, indicando que los precios relativos de la dieta habitual —de alto contenido calórico- están por debajo de los precios relativos de la dieta saludable (ver también las columnas 4 y 5 del cuadro 3). En estas circunstancias, los precios subvaluados de la dieta habitual para estas ciudades estarían señalando incentivos para el consumo de canastas de alto contenido calórico. En contraste, cuando este porcentaje es negativo se tendrá un menor acceso a dietas saludables, situación en la que a diciembre de 2010 se encontraron 23 de las 46 ciudades estudiadas. La subvaluación de la dieta habitual identifica entonces el porcentaje en el que se espera que el precio de estos productos suba para llegar a un equilibrio.

En las ciudades con márgenes de subvaluación y, sobre todo, en las que se alejan más del equilibrio (negativamente) como Ciudad Acuña, San Andrés Tuxtla, Tijuana y Puebla, si se buscara dar mayor acceso a la población a canastas de productos saludables, los gobiernos federal, estatal y municipal deberían procurar, por ejemplo, establecer medidas de política que reduzcan el precio de los ingredientes que conforman esta canasta saludable.

\section{El comportamiento temporal del Nut3-CiO}

Aun cuando el margen de sobrevaluación nutricional presentado en el cuadro 3 corresponde solo a diciembre de 2010, el Nut3- $\mathrm{CiO}_{\mathrm{j}, \mathrm{t}}$ no es estático, tiene una variación mensual y es distinto para cada una de las 46 ciudades a través del tiempo. La figura 1 del material suplementario muestra que la distribución cuantílica de este índice para cada mes en la muestra registra una ciclicidad, lo que sugiere la presencia de estacionalidad. También revela una volatilidad muy alta de los precios. De acuerdo a lo que indica la mediana y el tercer cuartil, en general se observa una subvaluación de la dieta habitual en la mayor parte de los meses investigados. Esto quiere decir que la mayoría de las ciudades de la muestra caen históricamente en una situación de subvaluación, donde la ca- nasta habitual resulta relativamente más barata que la dieta saludable.

\section{DISCUSIÓN}

Si bien varios estudios publicados han buscado determinar si los precios de alimentos de alto contenido calórico son más baratos que los alimentos saludables, ninguno ha proporcionado un indicador oportuno que provea información periódica confiable sobre los precios calóricos de dietas saludables y de canastas de consumo habitual para las ciudades al interior de un país (5-7). El Índice de Paridad Poder de Compra Nutricional (Nut3-CiO), introducido en el presente trabajo, contribuye a la investigación sobre la obesidad permitiendo localizar de manera específica, para varios meses y años, las ciudades, regiones e incluso países donde resulta más accesible (en términos de precios) llevar una dieta habitual o una dieta saludable. Adicionalmente, este nuevo instrumento arroja la brecha porcentual de precios entre los dos tipos de dietas, sugiriendo cuánto deberían subir —o bajar- los precios de la dieta habitual —o saludable - en una determinada ciudad para logar el equilibrio de precios.

El Nut3-CiO requiere que el analista defina las canastas alimentarias (dietas) de su interés (p. ej. alimentos saludables versus no saludables, o enteros versus procesados), que determine los ponderadores calóricos y que elija una ciudad numeraria de referencia. Las canastas de alimentos pueden formarse en base a características demográficas, sociales y económicas de la población objetivo, por ejemplo edad, género, estado de salud, escolaridad e ingreso. También podrían obtenerse los consumos calóricos por ciudad, en lugar de suponer que el promedio calórico nacional es el mismo para todas las regiones del país. Estos atributos otorgan al índice Nut3-CiO una flexibilidad altísima porque hacen posible examinar a distintos grupos de población de varias ciudades, a través de un período de tiempo y en función de características muy diversas.

Para determinar los índices de precios nutricionales asociados a la dieta saludable, la aplicación en México se basó en la recomendación alimentaria propuesta por Pérez Lizaur y Marván Laborde (13). Existen sin embargo una gama de aplicaciones del índice Nut3-CiO que van desde elegir alimentos específicos, y las ponderaciones de la canasta alimentaria, hasta recomendaciones nutricionales oficiales nacionales e internacionales. Por su parte, para determinar el perfil de consumo habitual de los mexicanos se utilizó la Encuesta Nacional de Ingresos y Gastos de los Hogares 2008, aunque hay otras alternativas como por ejemplo las hojas de balance alimentario (HBA) de la Organización para la Alimentación y la Agricultura, que también permiten generar índices de precios calóricos como los propuestos en este trabajo (17).

Los índices de precios calóricos aquí obtenidos son en sí mismos un concepto novedoso. La propuesta disponible que más se les acerca es el Índice Mundial de los Precios de los Alimentos de la Organización para la Agricultura y la Alimentación, que da seguimiento a una canasta de precios calóricos sobre productos de consumo básico representativos internacionales -aunque lo hace precisamente a partir de las $\operatorname{HBA}(10,17)$. Lamentablemente, publica datos mensuales pero no hace estimaciones por regiones al interior de los países.

El índice Nut3-CiO se relaciona con un amplia serie de aplicaciones de la PPC a distintos productos como la hamburguesa y sus ingredientes, productos agrícolas y forestales, precios inmobiliarios e incluso timbres postales (18-23). Vale señalar que hasta ahora no se había aplicado la PPC regional al estudio de dietas saludables versus dietas habituales.

Una limitación del índice Nut3-CiO es su sensibilidad a la calidad de los componentes, entre ellos el perfil calórico (ponderaciones) de la dieta habitual y de la dieta saludable, así como la elección de la ciudad numeraria. La construcción del índice también requiere que los precios de los alimentos elegidos en las dietas sean publicados periódicamente por el proveedor de precios, lo que puede limitar la construcción óptima de las dietas. Cualquier cambio de metodología en la elaboración de precios por parte del proveedor, o su decisión de no publicar por cualquier razón los precios de los alimentos incluidos en cualquiera de las dietas definidas en este estudio, podría poner en riesgo la continuidad de las mediciones. Por último, los precios calóricos construidos a partir de las contribuciones energéticas de cada alimento no son directamente comparables con el INPC y la CBA, debido a que estos 
índices están ponderados por el gasto nominal y no por el consumo en calorías de cada alimento.

\section{Conclusiones y recomendaciones}

El índice Nut3-CiO, introducido en el presente artículo, permite comparar periódicamente el diferencial de precios de dos tipos de dietas - habituales y saludables - entre las ciudades de un mismo país. Constituye un instrumento que facilita a las autoridades de salud identificar las ciudades en donde resulta más —o menos- fácil para los consumidores acceder a una dieta habitual o saludable en términos de costo. Asimismo, hace posible estimar el porcentaje de ajuste que deberían tener los precios re- lativos en cada ciudad para alcanzar los niveles de paridad de poder de compra nutricional.

La aplicación de este índice en México reveló que en general los precios relativos de la dieta habitual en este país son históricamente más bajos que los precios de la dieta saludable. Si bien es probable que tal diferencial de precios explique los altos niveles de sobrepeso y obesidad que se registran en este país, tal relación —así como el estudio de los vínculos entre el Nut3-CiO y diversas variables socioeconómicas y nutricionales- no pasan de ser hipótesis que podrían y deberían examinarse en estudios futuros.

Por último, queda demostrado que el índice $\mathrm{Nut}_{3}-\mathrm{CiO}$ es un instrumento de diagnóstico confiable y oportuno que

\section{REFERENCIAS}

1. World Health Organization. Obesity and Overweight: a WHO Report. 2011. Fact sheet No. 311. Disponible en: http://www.who. int/mediacentre/factsheets/fs311/en/ Acceso el 21 de agosto de 2011.

2. Sassi F. Obesity and the Economics of Prevention: Fit not Fat. París, Francia: OECD; 2010.

3. Lakdawalla D, Philipson T. The Growth of Obesity and Technological Change: A Theoretical and Empirical Examination. Working Paper No. 8946. The National Bureau of Economic Research. 2002.

4. Bleich S, Cutler D, Murray C, Adams A. Why is the Developed World Obese? Working Paper No. 12954. The National Bureau of Economic Research. 2007.

5. Drewnowski A, Monsivais P, Maillot M, Darmon N. Low-energy-density diets are associated with higher diet quality and higher diet costs in French adults. J Am Diet Assoc. 2007;107:1028-32.

6. Monsivais P, Drewnowski A. The rising cost of low-energy density foods. J Am Diet Assoc. 2007;107(12):2071-6.

7. Williams PG, Hull A, Kontos M. Trends in the affordability of the Illawarra Healthy Food Basket 2000-2007. Nutrition and Dietetics. 2009;66(1):27-32.

8. Fulgoni VL, Keast DR, Drewnowski A. Development and Validation of the Nutrient-Rich Foods Index: A Tool to Measure Nutritional Quality of Foods. The Journal of Nutrition. 2009;139:1549-54.

9. Drewnowski A. The Nutrient Rich Foods Index helps to identify healthy, affordable foods. Am J Clin Nutr. 2010;91(4):1095-101.
10. Organización de las Naciones Unidas para la Alimentación y la Agricultura (FAO). Perspectivas alimentarias, análisis de los mercados mundiales. Sistema mundial de información y alerta sobre la agricultura y la alimentación (SMIA). Roma, Italia: FAO; 2009. Disponible en: http://www.fao.org/giews/spanish/ fo/index.htm Acceso el 14 de septiembre de 2011.

11. Madrigal-Fritsch H, Pérez Gil-Romo SE, Romero-Ibarrola G, Batrouni-Kerkebe L, Domínguez-Cherit L, Fetter I, et al. Validación de indicadores cualitativos de alimentación: Escala de Guttman vs dieta habitual. Salud Publica Mex. 1993;35(2):194-201.

12. Rogoff K. The Purchasing Power Parity Puzzle. J Econ Lit. 1996;34:647-68.

13. Pérez Lizaur AB, Marván Laborde L. Manual de dietas normales y terapéuticas. $5{ }^{a}$ ed.

14. Ledesma Lozano JA, Chávez Villasana A, Pérez-Gil Romo F, Mendoza Martínez E, Calvo Carrillo C. Composición de alimentos Miriam Muñoz de Chávez. Valor nutritivo de los alimentos de mayor consumo. 2. ed México: McGraw Hill Interamericana; 2010.

15. Bourges-Rodríguez H, Casanueva E, Rosado JL. Recomendaciones de ingestión de nutrimentos para la población mexicana. México, D.F.: Editorial Médica Panamericana; 2009.

16. Balassa $B$. The purchasing-power parity doctrine: a reappraisal. J Polit Econ. 1964;72(6): 584-96.

17. Organización de las Naciones Unidas para la Alimentación y la Agricultura. Hoja de balance de alimentos. FAOSTAT. 2011. DisMéxico: Editorial Prensa Médica; 2005. permite orientar mejor el diseño de políticas públicas que estimulen y faciliten el acceso a dietas saludables. Su aplicación, que en el presente estudio se enfocó en México, puede sin dudas extenderse fácilmente a otros países de América Latina y el Caribe donde el sobrepeso y la obesidad son problemas de dimensiones preocupantes.

Agradecimiento. El autor quiere agradecer la eficiente asistencia de Margarito X. Reyes Ruiz, así como los valiosos comentarios de María de Lourdes Silva Fernández y Mónica Orduña Sosa al manuscrito del presente trabajo. Este proyecto fue apoyado por el fondo de investigación de la Universidad Popular Autónoma del Estado de Puebla, México. ponible en: http:// faostat.fao.org/DesktopDefault.aspx?PageID=354\&lang=es Acceso el 14 de septiembre de 2011.

18. Click RW. Contrarian MacParity. Economics Letters. 1996;53(2):209-12.

19. Cumby RE. Forecasting exchange rates and relative prices with the hamburger standard: is what you want what you get with McParity? Working Paper No. 5675. The National Bureau of Economic Research. 1996.

20. Susanto D, Parr Rosson C, Adcock FJ. Market integration and convergence to the law of one price in the North American onion markets. Agribusiness. 2008;24(2):177-91.

21. Nanang DM. A multivariate cointegration test of the law of one price for Canadian softwood lumber markets. Forest Policy and Economics. 2000;1(3-4):347-55.

22. Clark S, Coggin T. Trends, Cycles and Convergence in U.S. Regional House Prices. The Journal of Real Estate Finance and Economics. 2009;39(3):264-83.

23. Maesepp MN. Postage Price Parity. Economic Papers: A journal of applied economics and policy. 2009;28(2):169-75.

Manuscrito recibido el 4 de noviembre de 2010. Aceptado para publicación, tras revisión, el 30 de junio de 2011. 
ABSTRACT Objective. Develop the Index of Nutritional Purchasing Power Parity (Nut3-CiO) as an instrument to compare the caloric costs of a healthy versus an unhealthy diet between regions or cities in a country over time.

Index of Nutritional Purchasing Power Parity: comparison of caloric costs of a healthy versus an unhealthy diet

Methods. Indices of caloric prices were constructed and the "law of one price" was used to derive the Nut3-CiO index. Caloric inflation rates were obtained using basic descriptive statistics. The Nut3-CiO was applied in the major cities of Mexico during the period from January 1996 to December 2010.

Results. The statistical behavior of the Nut3-CiO revealed that, in Mexican cities, products for a typical diet are less expensive than products for a healthy diet. The findings showed a cyclical behavior to the index, a high correlation between inflation for the typical diet and inflation for the market basket, and a high persistence of prices.

Conclusions. The Nut3-CiO index makes it possible to periodically compare the price differential of two types of diets-typical and healthy-between cities in a single country. This instrument could help health authorities identify the cities where it is easier or more difficult for consumers to access a typical or healthy diet in terms of cost. Furthermore, it makes it possible to estimate the percentage adjustment necessary in each city to attain levels of nutritional purchasing power parity.

Key words Obesity; overweight; diet; feeding behavior; food; health economics; economic indexes; biostatistics; Mexico. 
Material suplementario del artículo: Mendoza Velázquez A. Índice de Paridad Poder de Compra Nutricional: comparación de los precios calóricos entre dietas habituales y saludables. Rev Panam Salud Publica. 2012;31(1)17-24.

CUADRO 1. Perfil del consumo calórico por producto de los mexicanos ${ }^{\mathrm{a}}$

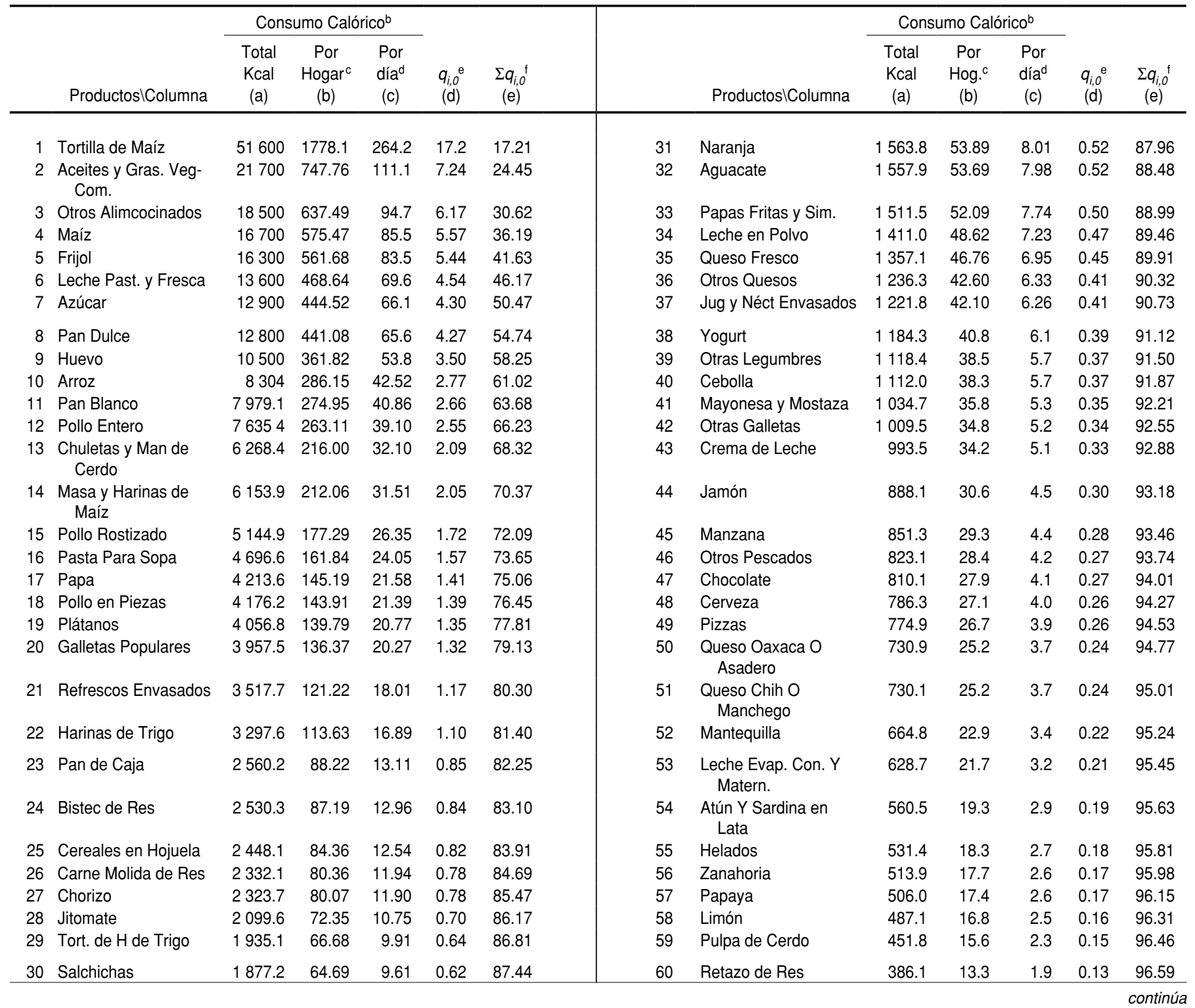


CUADRO 1. Continuación

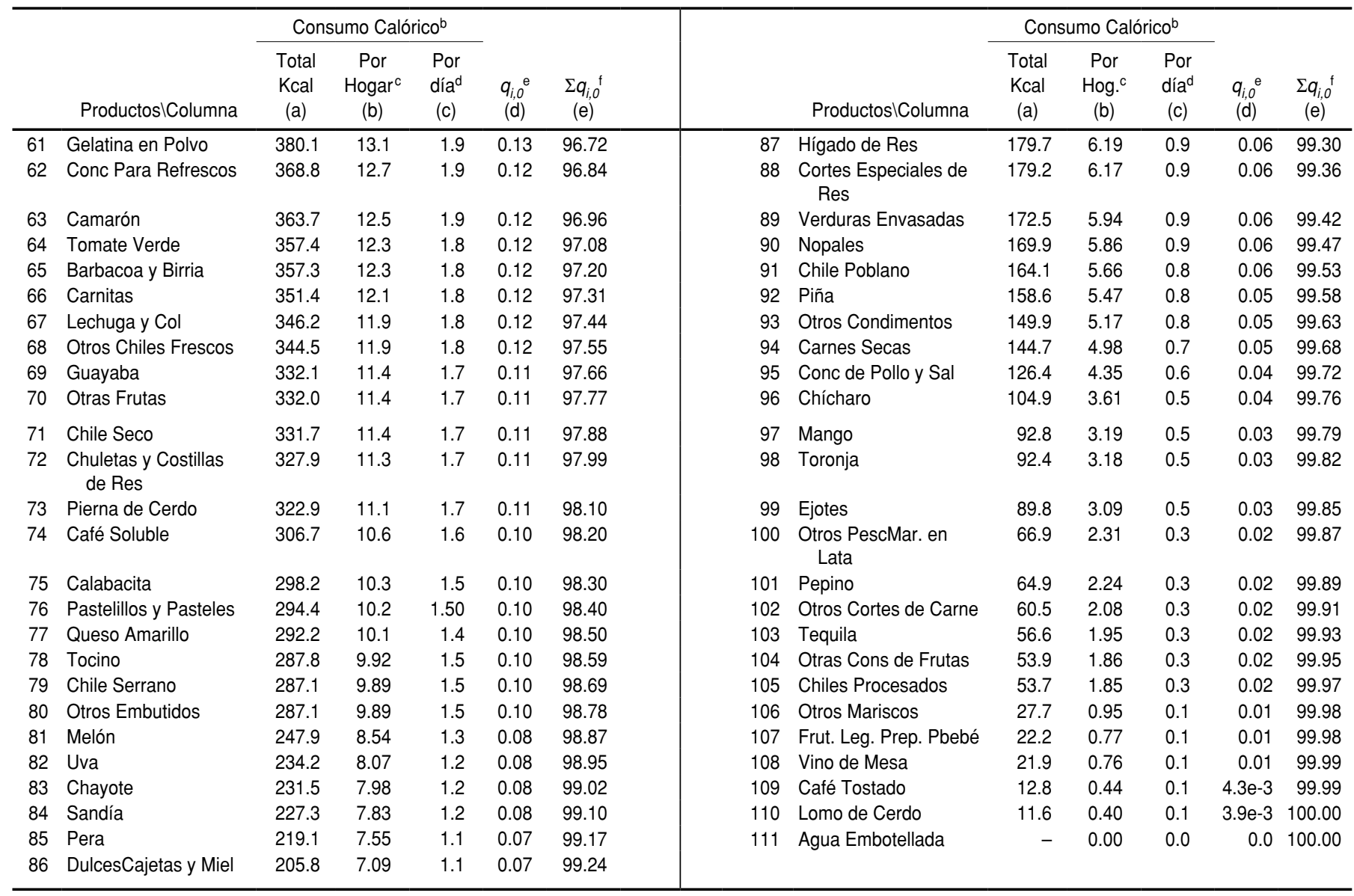

a En base a la Encuesta Nacional de Ingreso y Gasto de los Hogares en México 2008 (ENIGH).

b La transformación de consumo de los mexicanos de unidades originales ( $\mathrm{kg}$, litros, etc.) a kilocalorías (Kcal) se basa en la información provista en el Cuadro 2 de este material suplementario.

c Se refiere al consumo calórico de cada uno de los 29,468 hogares en México en los días de la encuesta.

d El consumo diario calórico individual se obtiene dividiendo el total de calorías por hogar entre el número de días en la encuesta.

${ }^{\text {e }} q_{i, 0}$ Se refiere a la participación calórica porcentual de cada alimento respecto al total de calorías.

${ }^{f} \Sigma q_{i, 0}$ es la participación calórica acumulada de perfil de consumo del mexicano. 


\begin{tabular}{|c|c|c|c|c|c|c|c|c|c|c|c|c|c|}
\hline 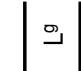 & 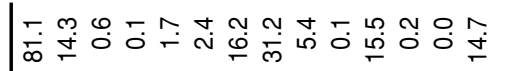 & $\stackrel{m}{m}$ & 守菅 & 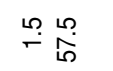 & $\overline{0} \underset{\mathrm{p}}{\mathrm{p}}$ & 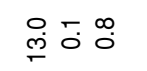 & 管 & 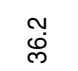 & ㄷㅇㅇ & $\stackrel{\infty}{\infty}$ & $\stackrel{m}{\circ}$ & 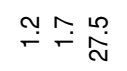 & \\
\hline $\bar{a}$ & 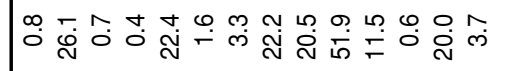 & $\stackrel{\rho}{\rho}$ & 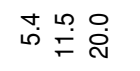 & 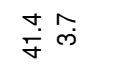 & $\stackrel{\leftrightarrow}{\circ} \stackrel{m}{\sim}$ & $\underset{+}{\stackrel{0}{o}} \hat{0} \stackrel{\infty}{0}$ & $\begin{array}{ll}\infty \\
\dot{\sigma} \\
\dot{0}\end{array}$ & $\frac{\stackrel{+}{+}}{\stackrel{2}{4}}$ & $\stackrel{\infty}{\circ} \hat{i}$ & $\stackrel{\leftrightarrow}{\stackrel{\leftrightarrow}{N}}$ & $\stackrel{\infty}{\circ}$ & 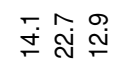 & \\
\hline @ & 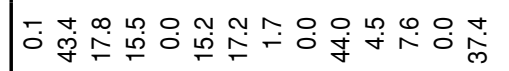 & 号 & 吕 & 용요 & $\because \infty$ & 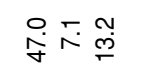 & $\stackrel{n}{\circ} \underset{\infty}{0} \stackrel{0}{0}$ & $\because$ & 숭ㅇㅇㅇ & $\stackrel{\infty}{+}$ & $\stackrel{n}{\stackrel{q}{q}}$ & & \\
\hline ㄴ. & 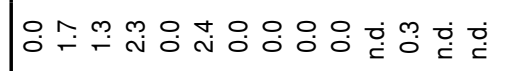 & $\stackrel{\circ}{\circ}$ & $\stackrel{m}{\dot{\sigma}} \underset{\dot{C}}{\dot{0}} \stackrel{0}{0}$ & $\because \circ$ & $\stackrel{\circ}{\circ} \stackrel{0}{\circ}$ & 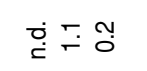 & $\because 0$ & $\stackrel{\circ}{\circ}$ & $\stackrel{\circ}{\circ}$ & $\stackrel{\circ}{\circ}$ & $\stackrel{2}{\circ}$ & $\stackrel{\circ}{\circ}$ & \\
\hline$\stackrel{\circ}{I}$ & 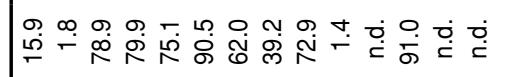 & $\bar{\sigma}$ & 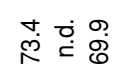 & 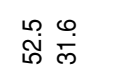 & 怨 & 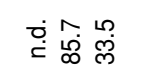 & $\begin{array}{l}m \\
\stackrel{m}{6} \stackrel{\infty}{\infty} \stackrel{\infty}{\infty}\end{array}$ & 孞 & $\infty$ & 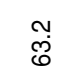 & i & & \\
\hline$\stackrel{\rho}{\longrightarrow}$ & 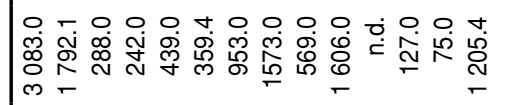 & 品 & 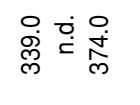 & 웅 & 웅요 & 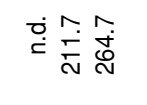 & 잃 芦 & 家 & 悹 & 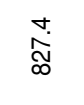 & $\stackrel{\infty}{\underset{i}{0}}$ & 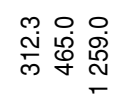 & \\
\hline 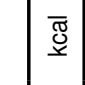 & 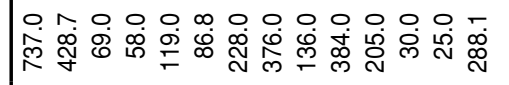 & $\begin{array}{l}\infty \\
\grave{\infty} \\
\stackrel{\infty}{\infty}\end{array}$ & 이 융영 & 余 & 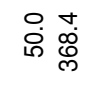 & 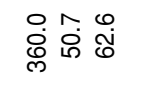 & 官㐫 & $\stackrel{\circ}{\stackrel{\infty}{\infty}}$ & 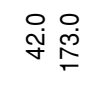 & $\stackrel{\substack{0 \\
\infty}}{\stackrel{\infty}{\sigma}}$ & $\begin{array}{l}\stackrel{\circ}{\circ} \\
\stackrel{\infty}{\circ}\end{array}$ & 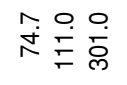 & 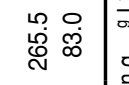 \\
\hline $\begin{array}{l}\stackrel{\circ}{0} \\
\stackrel{0}{0} \\
\frac{0}{0}\end{array}$ & 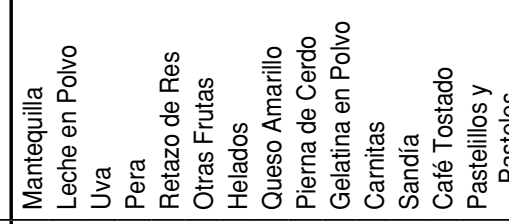 & 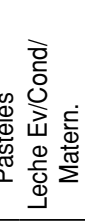 & 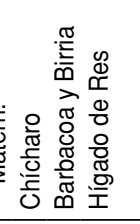 & 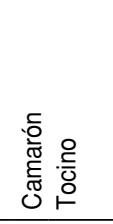 & 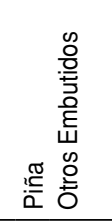 & 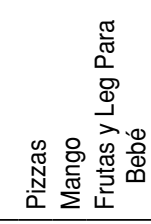 & 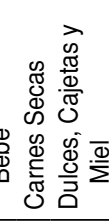 & 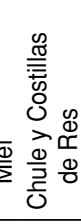 & 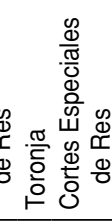 & 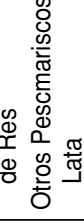 & 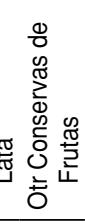 & 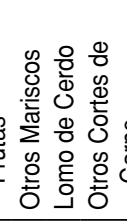 & \\
\hline 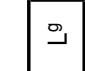 & 㐘 & $\stackrel{n}{\dddot{O}}$ & 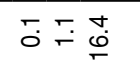 & 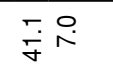 & $\bar{\sigma}$ & 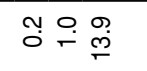 & $\stackrel{\infty}{\infty}$ & $\stackrel{+}{+\infty}$ & $\stackrel{m}{\infty} \stackrel{0}{+}$ & $\stackrel{\circ}{\circ}$ & $\stackrel{n}{N}$ & & \\
\hline $\bar{a}$ & 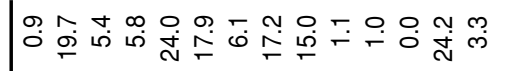 & $\stackrel{\circ}{\circ}$ & 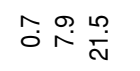 & i & $\stackrel{\circ}{\circ}$ & $\stackrel{\circ}{\circ} \stackrel{\circ}{\circ} \stackrel{\infty}{\sim}$ & & $\stackrel{\circ}{\circ}$ & & $\stackrel{m}{i}$ & 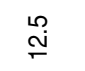 & jị & \\
\hline 온 & 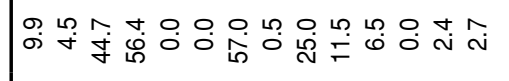 & $\stackrel{m}{\infty}$ & மீ요용 & $\stackrel{\sim}{\stackrel{\dot{\gamma}}{\gamma}}$ & $\stackrel{\infty}{\infty} \underset{0}{\infty}$ & 疍 & 兽 & $\stackrel{m}{i}$ & $\stackrel{\sim}{\circ} \stackrel{9}{\leftrightarrow}$ & $\stackrel{\circ}{\circ}$ & $\overline{\dot{\theta}}$ & & \\
\hline ¿ & 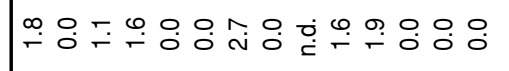 & $\stackrel{\infty}{\circ}$ & $\stackrel{\operatorname{Ln}}{\infty} \stackrel{\infty}{\stackrel{\infty}{0}} \stackrel{0}{0}$ & $\stackrel{0}{0} \stackrel{0}{0}$ & 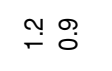 & $\ddot{O} \dot{0} \overline{\mathrm{N}}$ & $\stackrel{\infty}{\circ} \stackrel{?}{\sim}$ & $\stackrel{\infty}{\sim}$ & m. & $\stackrel{\circ}{\circ}$ & $\stackrel{9}{0}$ & & \\
\hline$\stackrel{\circ}{1}$ & 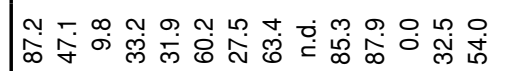 & $\underset{\infty}{\bar{\infty}}$ & $\bar{\delta} \stackrel{\circ}{\circ} \stackrel{n}{i} \stackrel{0}{i}$ & & ז̊ & 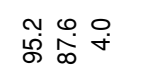 & $+R$ & $\underset{\infty}{\infty}$ & & $\stackrel{\substack{++}}{2}$ & $\stackrel{\overbrace{}}{\check{F}}$ & & \\
\hline$\stackrel{?}{\stackrel{2}{s}}$ & 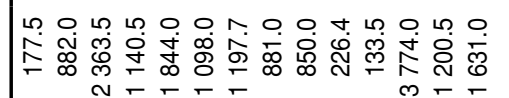 & 品 & 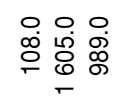 & $\begin{array}{l}0 \\
0 \\
\infty \\
0 \\
0\end{array}$ & 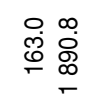 & 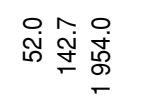 & 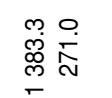 & 員 & & 吕 & $\underset{亡}{\stackrel{\Gamma}{i}}$ & & \\
\hline 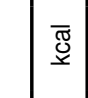 & 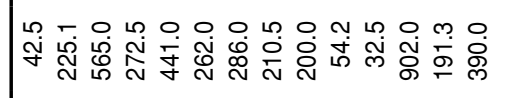 & $\stackrel{\circ}{9}$ & 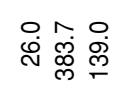 & 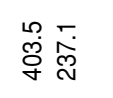 & 啇 & 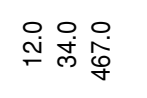 & $\stackrel{f}{f}$ & 灾 & & 임 & 离 & & \\
\hline בְ & 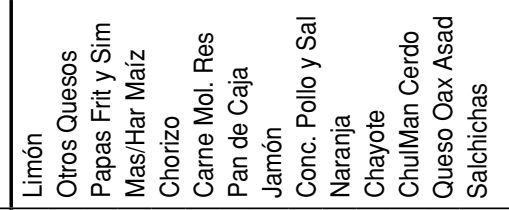 & 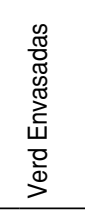 & 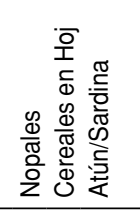 & 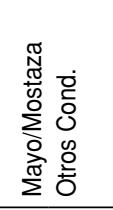 & $\begin{array}{l}\frac{0}{\pi} \\
\frac{\pi}{\pi} \\
\frac{\pi}{0} \\
\frac{0}{0} \frac{0}{0} \\
0\end{array}$ & 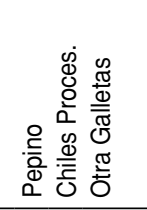 & 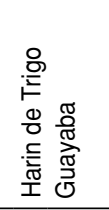 & $\frac{\sqrt[N]{N}}{\sum}$ & 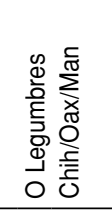 & $\begin{array}{l}\text { 윰 } \\
0 \\
0 \\
0 \\
\frac{0}{0} \\
\frac{0}{3} \\
\frac{0}{2}\end{array}$ & 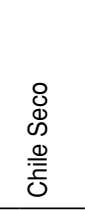 & 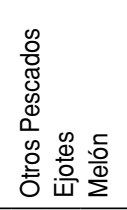 & $\begin{array}{l}\frac{0}{0} \\
\frac{0}{0} \\
\frac{0}{\frac{7}{0}}\end{array}$ \\
\hline 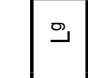 & 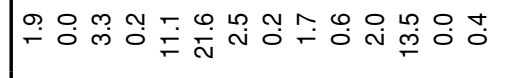 & $\ddot{g}$ & $\stackrel{\infty}{+\infty} \stackrel{\substack{N \\
\sim}}{\substack{\infty \\
\infty}}$ & 00 & 웅 & ণ̃ & ִָ & $\stackrel{\circ}{\circ}$ & ச̆ & $\stackrel{2}{\sim}$ & $\stackrel{\leftrightarrow}{+}$ & $\stackrel{m}{\stackrel{9}{\stackrel{P}{\sim}}}$ & \\
\hline $\bar{a}$ & 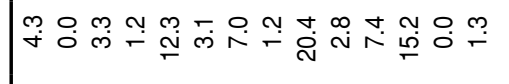 & $\begin{array}{l}\stackrel{\infty}{\infty} \\
\stackrel{\infty}{\leftarrow}\end{array}$ & 或 品 $\stackrel{0}{亡}$ & & $\sim 0^{\circ}$ & $\stackrel{\circ}{\circ} \stackrel{0}{\leftarrow} \stackrel{\mathrm{N}}{\mathrm{N}}$ & זִ & 0 & & $\stackrel{\circ}{\circ}$ & $\stackrel{m}{\infty}$ & & \\
\hline 오 & 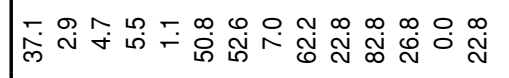 & 웅 & $\stackrel{\infty}{L_{\infty}} \stackrel{\circ}{0} \overline{0}$ & $\stackrel{0}{\mathscr{\infty}}$ & $\leqslant 0$ & 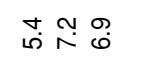 & & $\frac{d}{8}$ & & $\check{F}$ & 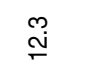 & $\hat{\emptyset}$ & \\
\hline ¿ & 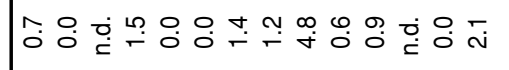 & $\because$ & 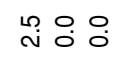 & $0 N$ & No & 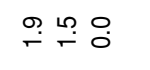 & & 0 & & $\stackrel{\text { ì }}{9}$ & $\stackrel{\circ}{\circ}$ & & \\
\hline$\stackrel{\circ}{I}$ & 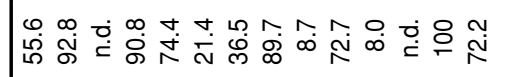 & 官 & 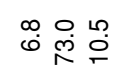 & & t & $\underset{\infty}{\infty} \underset{\infty}{\infty} \underset{\infty}{\infty} \stackrel{-}{\infty}$ & & 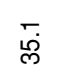 & & $\infty$ & 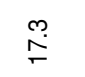 & & \\
\hline 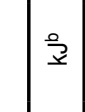 & 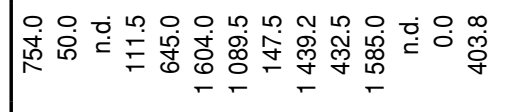 & $\begin{array}{l}\circ \\
\infty \\
\infty\end{array}$ & 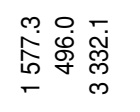 & & $\underset{\substack{L \\
\stackrel{\rho}{\rho} \\
\infty}}{\infty}$ & $\begin{array}{lll}0 & 0 & 0 \\
0 & 0 & 0 \\
0 & 0\end{array}$ & & $\stackrel{\circ}{\infty}$ & & 呙 & 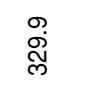 & & \\
\hline$\overline{\widetilde{\widetilde{d}}}$ & 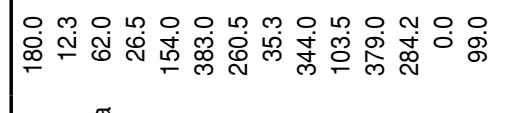 & 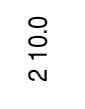 & 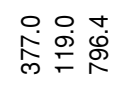 & 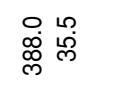 & 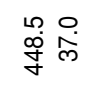 & 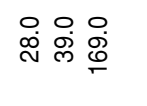 & & 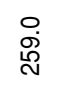 & 임 & $\begin{array}{l}\circ \\
\dot{g}\end{array}$ & $\frac{0}{\infty}$ & & \\
\hline 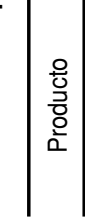 & 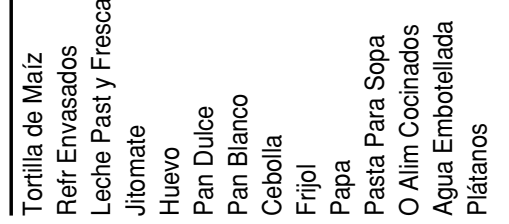 & 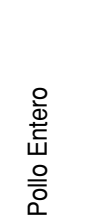 & 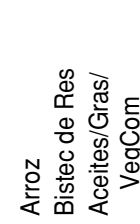 & & 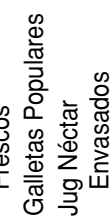 & 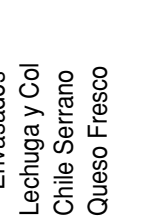 & $\frac{\pi}{\frac{\sigma}{0}}$ & 言 & 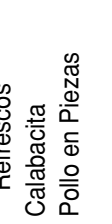 & $\begin{array}{l}\stackrel{\mathbb{N}}{\mathbb{N}} \\
\stackrel{N}{N} \\
\stackrel{\mathbb{W}}{\Sigma}\end{array}$ & 농 & 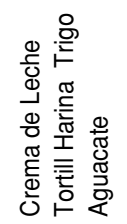 & $\begin{array}{l}\frac{0}{0} \\
\frac{3}{3} \\
\infty \\
\infty \\
0\end{array}$ \\
\hline
\end{tabular}




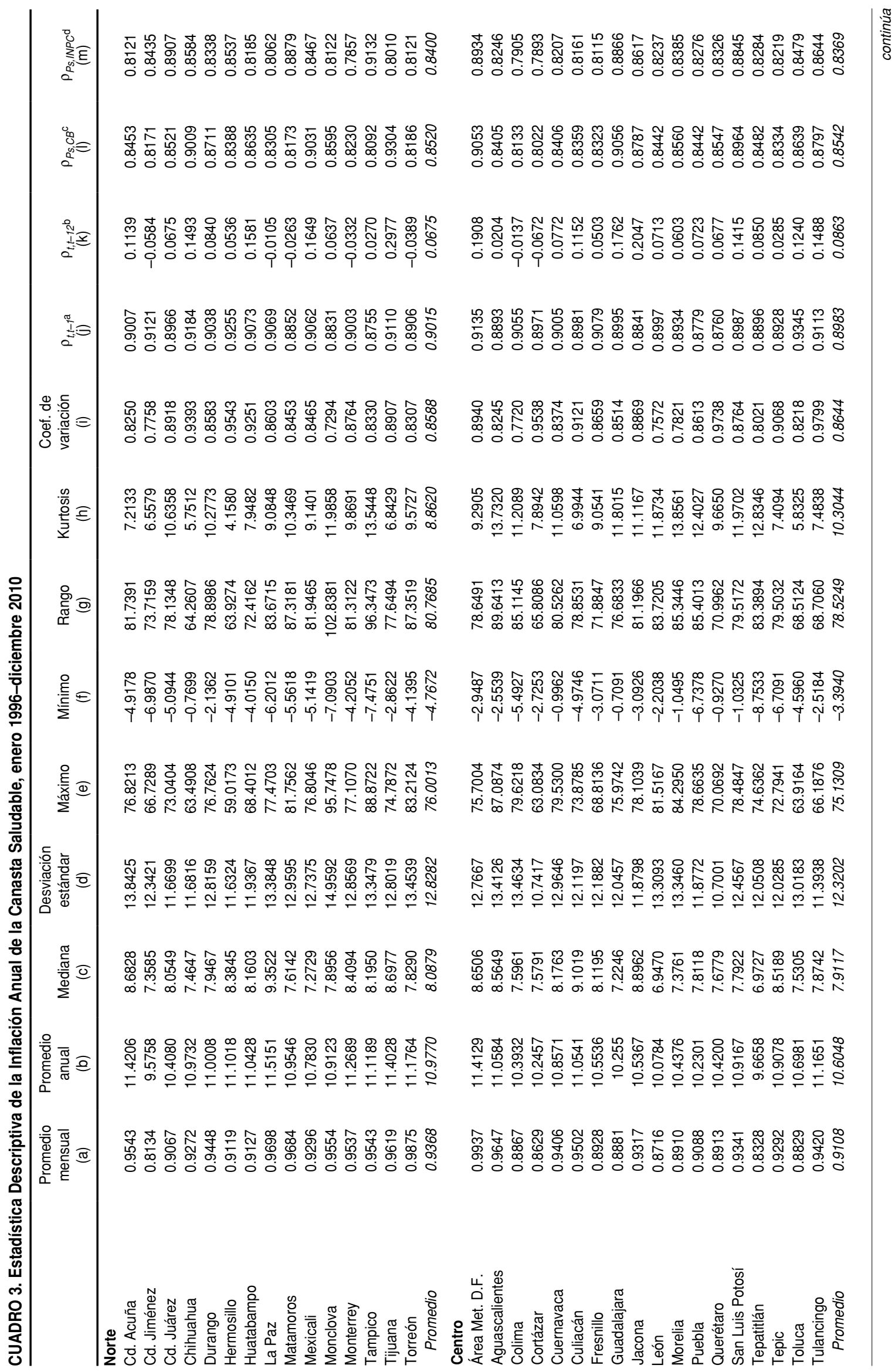




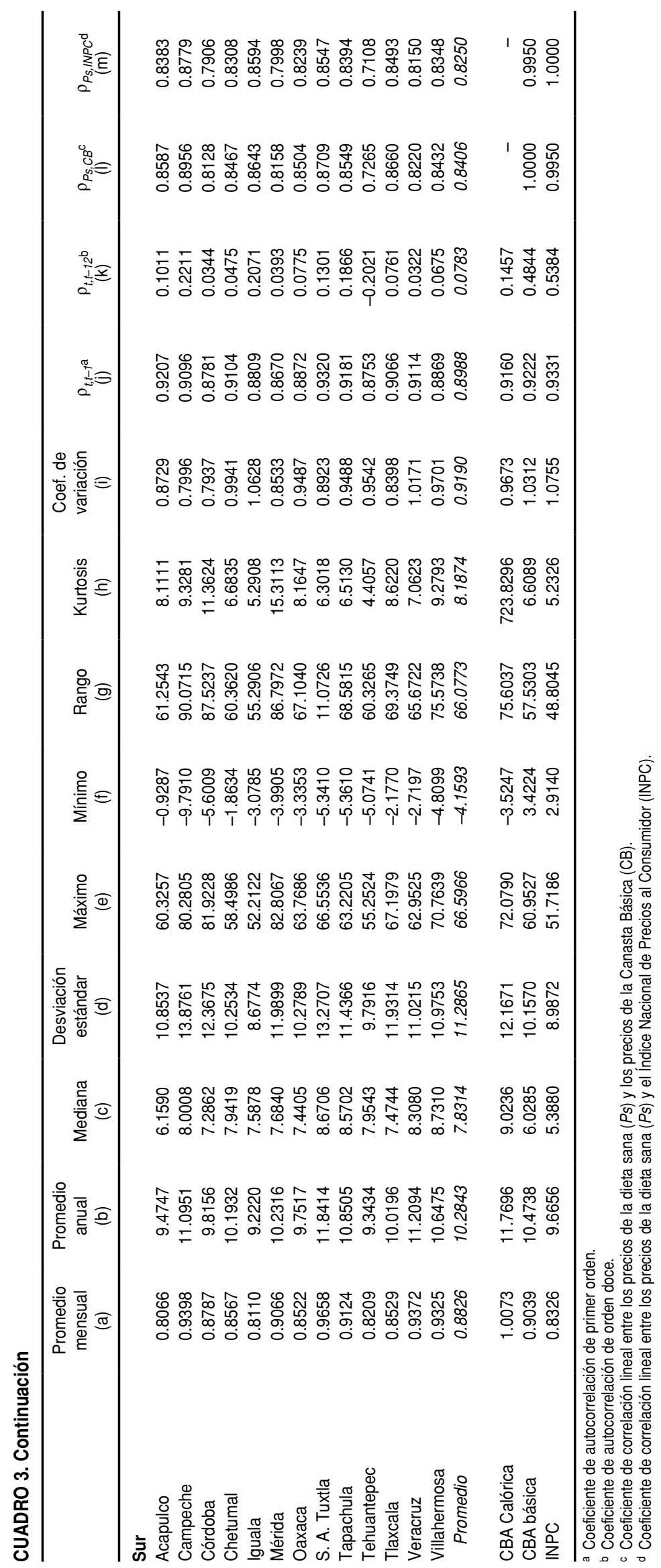




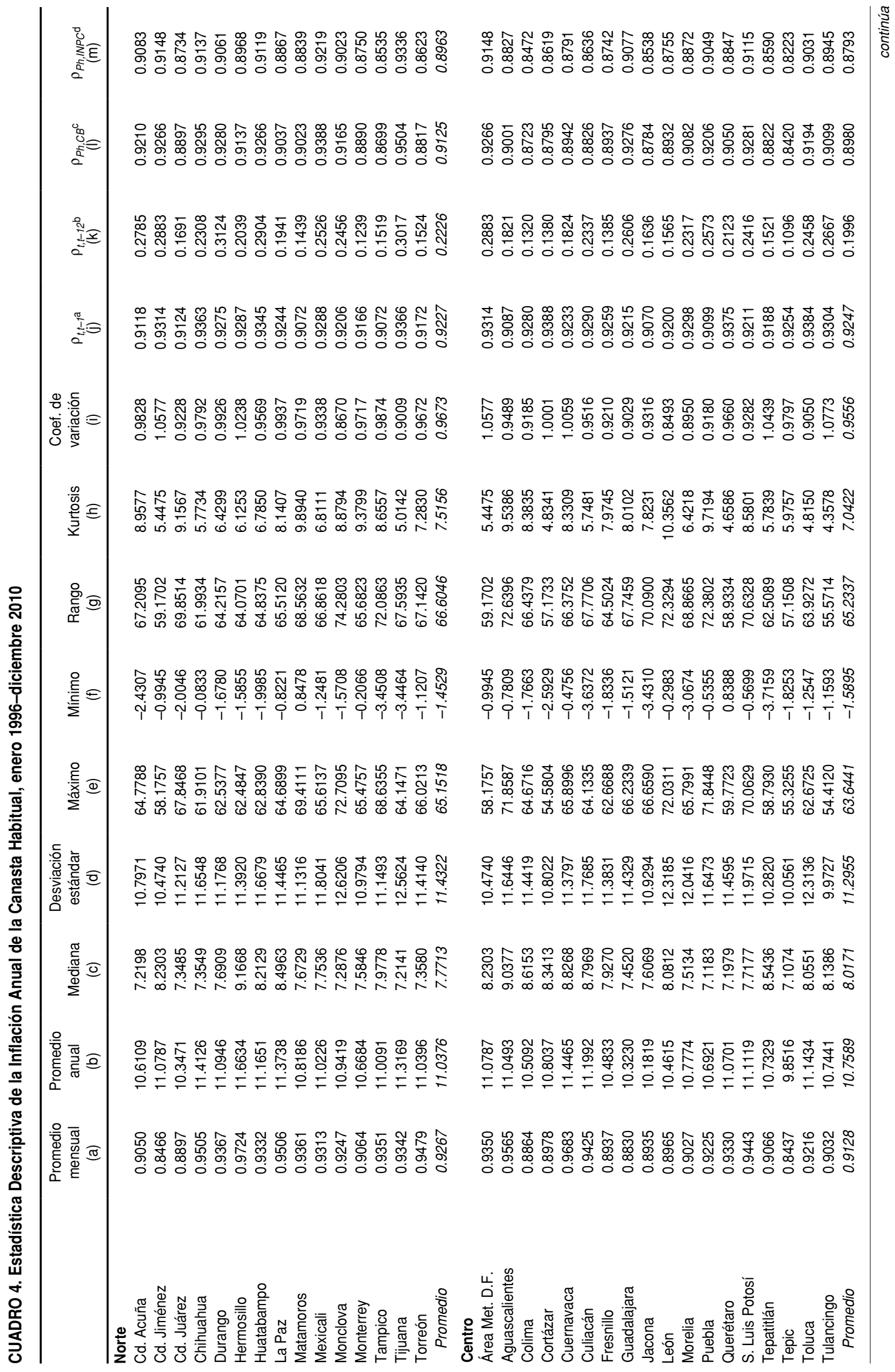




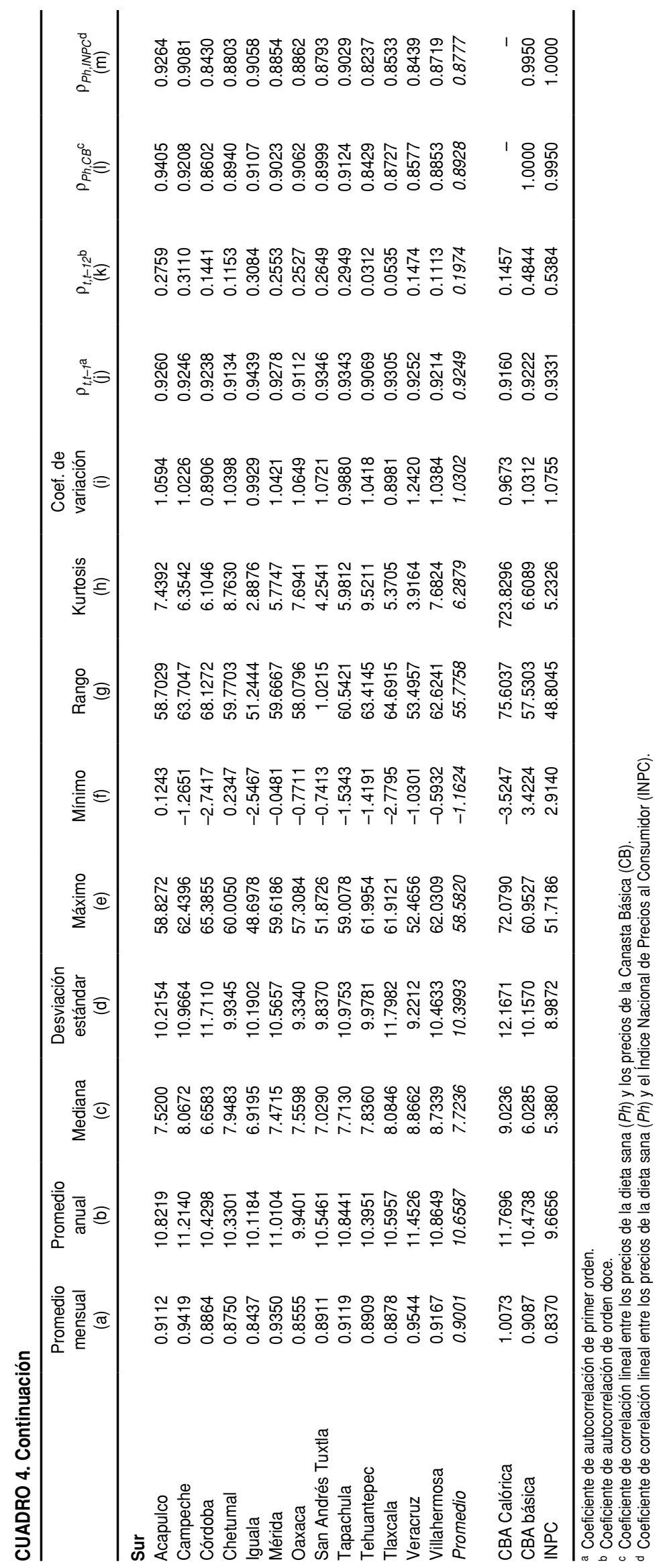


FIGURA 1. Índice de Paridad Poder de Compra Nutricional Nut3-CiO, México, enero 1996-diciembre 2010

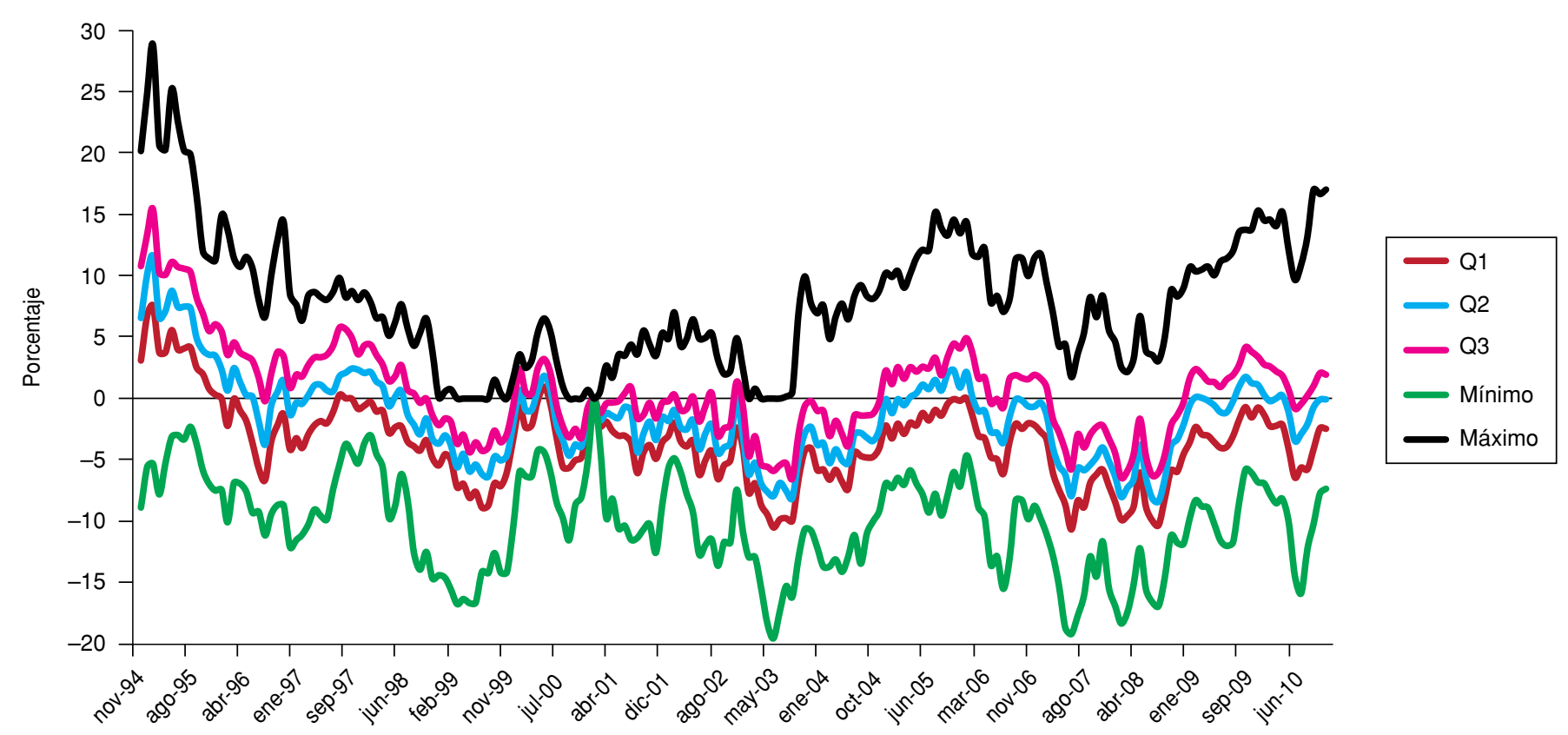

Fuentes: Elaboración del autor con base a los índices de precio Laspeyres. 